\title{
Recent highlights from STAR
}

\author{
Wangmei Zha ${ }^{1, \star}$ for the STAR Collaboration \\ ${ }^{1}$ Department of modern physics, University of Science and Technology of China, Hefei, 230026, China
}

\begin{abstract}
The Solenoidal Tracker at RHIC (STAR) experiment takes advantage of its excellent tracking and particle identification capabilities at mid-rapidity to explore the properties of strongly interacting QCD matter created in heavy-ion collisions at RHIC. The STAR collaboration presented 7 parallel and 2 plenary talks at Strangeness in Quark Matter 2017 and covered various topics including heavy flavor measurements, bulk observables, electro-magnetic probes and the upgrade program. This paper highlights some of the selected results.
\end{abstract}

\section{Introduction}

Relativistic heavy-ion collisions provide a unique tool to study the most intriguing aspects of the strong interaction in the laboratory. In high energy heavy-ion collisions performed at RHIC and the LHC, the achieved energy densities are extremely high so that a new form of matter, referred to as the quark-gluon plasma (QGP) [1], can be created. This matter has been observed to quickly thermalize and evolve as a perfect fluid with small viscosity over entropy ratio. The STAR heavy-ion program at top RHIC energies is aimed to quantitatively study the properties of the strongly-interacting QGP matter created at vanishingly small baryon density. While at finite baryon density from the Beam Energy Scan (BES) phase-I program at lower energies, the nature of the phase transition is being studied.

At Strangeness in Quark Matter 2017, the STAR collaboration has presented results from two recently installed detectors for heavy-flavor studies, the Heavy Flavor Tracker (HFT) and Muon Telescope Detector (MTD). The HFT, which facilitates topological reconstruction of open heavy flavor hadrons, has enabled the first measurement of $D^{0}$ azimuthal anisotropy at RHIC, which helps constrain the charm quark spatial diffusion coefficient in the QGP. The MTD, which enables identification and triggering of muons, opens the door to measure quarkonia via the di-muon decay channel at STAR, providing insights to the understanding of the dissociation and regeneration phenomena of quarkonia in the QGP. With the full BES phase-I datasets, the collectivity of medium has been systematically studied via directed, elliptic and higher order harmonics together with femtoscopy measurements. In non-central relativistic heavy-ion collisions, the created medium possesses strong vorticity, which causes the spin of particles to trend to align with the system's global angular momentum. The first differential measurement of the $\Lambda$ polarization as a function of $\Lambda$ azimuthal angle relative to the reaction plan has been reported at this conference. For the electro-magnetic probes, the first dielectron measurement at very low $p_{T}$ has been shown at this conference.

\footnotetext{
^e-mail: wangmei@ rcf.rhic.bnl.gov
} 

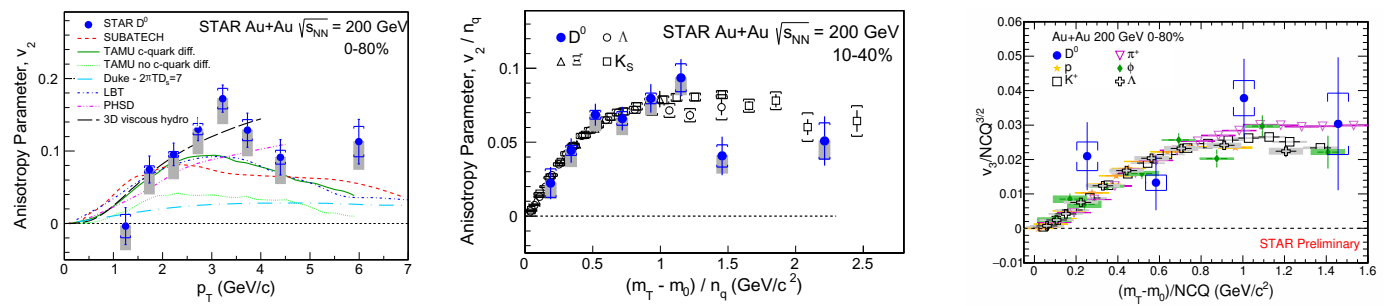

Figure 1. Left: $v_{2}$ as a function of $p_{T}$ for $\mathrm{D}^{0}$ mesons in $0-80 \%$ centrality [3]. Middle (right): $v_{2} / n_{q}$ in $10-40 \%$ centrality $\left(v_{3} /\left(n_{q}\right)^{3 / 2}\right.$ in $0-80 \%$ centrality) as a function of $\left(m_{T}-m_{0}\right) / n_{q}$ for $\mathrm{D}^{0}$ and light flavor hadrons [3].

\section{Heavy flavor measurements}

In heavy-ion collisions at RHIC, heavy flavor quarks are predominantly produced by initial hard parton scatterings, and subsequently experience the whole evolution of the system. Therefore they can serve as calibrated probes to study the properties of the strongly coupled Quark Gluon Plasma (sQGP). In particular, the anisotropic flow of open heavy flavor hadrons is sensitive to the interactions of heavy flavor quarks with the medium and could also help constrain the magnitude of their spatial diffusion coefficient $D_{s}$ in sQGP [2]. Shown in the left panel of Fig. 1 are the $v_{2}$ of $\mathrm{D}^{0}$ mesons as a function of $p_{T}$ in the $0-80 \%$ centrality interval [3]. The measured $v_{2}$ are compared to theoretical calculations [49], which use different approaches to describe the transportation or interaction of charm quarks in the medium. The hydrodynamic model [4] with parameters tuned to reproduce the light flavor hadron $v_{2}$ can reasonably describe the $D^{0} v_{2}$, indicating that charm quarks may have achieved full thermal equilibrium in these collisions. The transport models [6-9] with a temperature dependent diffusion coefficient in the range $2<2 \pi T D_{s}<12$ for $T_{c}<T<2 T_{c}$ are also able to reproduce the measured values. The left (middle) panel of Fig. 1 shows the $v_{2}\left(v_{3}\right)$ values scaled by the number of constituent quarks (NCQ, $\left.n_{q}\right)$ for $D^{0}$ mesons and a number of light flavor hadrons, as a fucntion of $\left(m_{T}-m_{0}\right) / n_{q}$. The scaled $v_{2}$ and $v_{3}$ for all the species including $D^{0}$ falls on the same trend, which also suggests that charm quarks flow with the medium.

The yield ratios of $D_{s}^{ \pm} / D^{0}$ and $\Lambda_{c}^{ \pm} / D^{0}$ in $\sqrt{s_{\mathrm{NN}}}=200 \mathrm{GeV} \mathrm{Au}+\mathrm{Au}$ collisions at RHIC are shown in Fig. 2. These measurements can help us to understand the hadronization mechanism of charm quarks in the QGP medium [10-12]. Both ratios show significant enhancements compared to those in $\mathrm{p}+\mathrm{p}$ collisions predicted by PYTHIA, suggesting that charm quarks could hadronize via the coalescence mechanism in the QGP. For $\Lambda_{c}^{ \pm} / D^{0}$, model calculations with coalescence of thermalized charm quarks in the QGP medium are compatible with data within uncertainties [10], while for $D_{s}^{ \pm} / D^{0}$ the TAMU model calculation for the $10-40 \%$ centrality interval with coalescence of charm quarks seems to under-predict the measured values [9].

Quarkonium dissociation by the color-screening effect in the hot and dense medium was initially proposed as signature of the QGP formation [13]. However, the interpretation of $J / \psi$ suppression observed in experiments has remained a challenge due to the regeneration from the coalescence of deconfined heavy quark pairs in the medium, the cold nuclear matter (CNM) effects and feed-down contributions. In order to quantify those effects, we present (i) measurements of nuclear modification factors for inclusive $J / \psi$ production in $\mathrm{p}+\mathrm{Au}$ collisions at $\sqrt{s_{\mathrm{NN}}}=200 \mathrm{GeV}$; (ii) the first measurement of the double ratio of inclusive $\psi(2 S)$ and $J / \psi$ production rates at mid-rapidity between $\mathrm{p}+\mathrm{p}$ and $\mathrm{p}+\mathrm{Au}$ collisions at $\sqrt{s_{\mathrm{NN}}}=200 \mathrm{GeV}$; and (iii)measurements of $\Upsilon$ nuclear modification factors in $\mathrm{Au}+\mathrm{Au}$ collisions at $\sqrt{s_{\mathrm{NN}}}=200 \mathrm{GeV}$. 


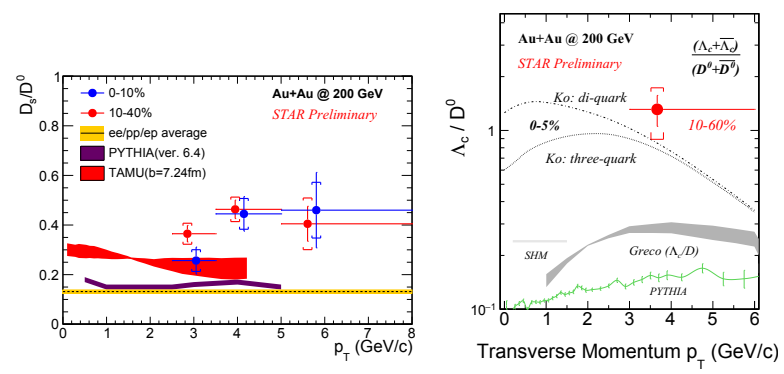

Figure 2. Yield ratios of $D_{s} / D^{0}$ in $0-10 \%$ and $10-40 \% \mathrm{Au}+\mathrm{Au}$ collisions (left) and $\Lambda_{c} / D^{0}$ in $10-60 \%$ central $\mathrm{Au}+\mathrm{Au}$ collisions (right) as a function of $p_{T}$.
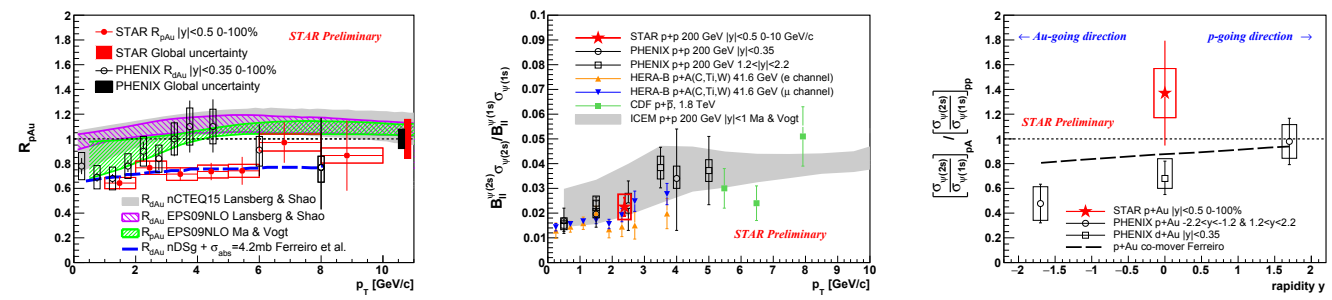

Figure 3. Left: nuclear modification factor $R_{p A u}$ as a function of $p_{T}$ for inclusive $J / \psi$ in the di-muon decay. Middle: ratio of $\psi(2 S)$ to $J / \psi$ production rates as a function of $p_{T}$. Right: double ratio of $\psi(2 S)$ to $J / \psi$ production rates as a function of rapidity in $\mathrm{p}+\mathrm{Au}$ and $\mathrm{d}+\mathrm{Au}$ collisions with respect to that in $\mathrm{p}+\mathrm{p}$ collisions..

Shown in the left panel of Fig. 3 is the $R_{p A u}$ of inclusive $J / \psi$ in p+Au collisions. The measured $R_{p A u}$ is generally consistent with the $R_{d A u}$ result reported by PHENIX [14] within uncertainties. The theoretical predictions with only the nuclear PDF effect using the nCTEQ15 [15] or EPS09NLO [16] nuclear PDFs sets can touch the upper limit of the data. However, the calculation including an additional nuclear absorption effect [17] is favored by the data. The middle panel of Fig. 3 shows the ratio of inclusive $J / \psi$ and $\psi(2 S)$ production cross sections as a function of $p_{T}$ in $\mathrm{p}+\mathrm{p}$ collisions at $\sqrt{s}=200 \mathrm{GeV}$. The measurement follows the trend of the world-wide data and could be described by the ICEM calculation [18]. Shown in the right panel of Fig. 3 is the double ratio of $\psi(2 S)$ and $J / \psi$ production rates between $\mathrm{p}+\mathrm{p}$ and $\mathrm{p}+\mathrm{Au}$ collisions as a function of rapidity. Taking into account the large uncertainties, the measurement is consistent with the published PHENIX results [19] and the co-mover model calculation [20].

The $R_{A A}$ of $\Upsilon(1 S)$ as a function of the number of participating nucleons $\left(N_{\text {part }}\right)$ is shown in the left panel of Fig. 4. The measurement is compared to that in $\mathrm{Pb}+\mathrm{Pb}$ collisions at $\sqrt{s_{\mathrm{NN}}}=2.76$ $\mathrm{TeV}$ from CMS [21] and they show similar suppression. Please note that the feed-down contributions from excited states are not excluded from the $\Upsilon(1 S) R_{A A}$. The STAR results are also compared to different theoretical model calculations [22-24], as shown in the middle panel of Fig. 4. Two limiting cases for the in-medium potential between the quark-antiquark pairs that are often used are the internal energy corresponding to a strongly bound scenario (SBS), and the free energy corresponding to a more weakly bound secnario (WBS). Our data seem to favor the SBS scenario toward central collisions. The $p_{T}$ dependence of $\Upsilon(1 S) R_{A A}$ from STAR is shown in the right panel of Fig. 4 and also compared 

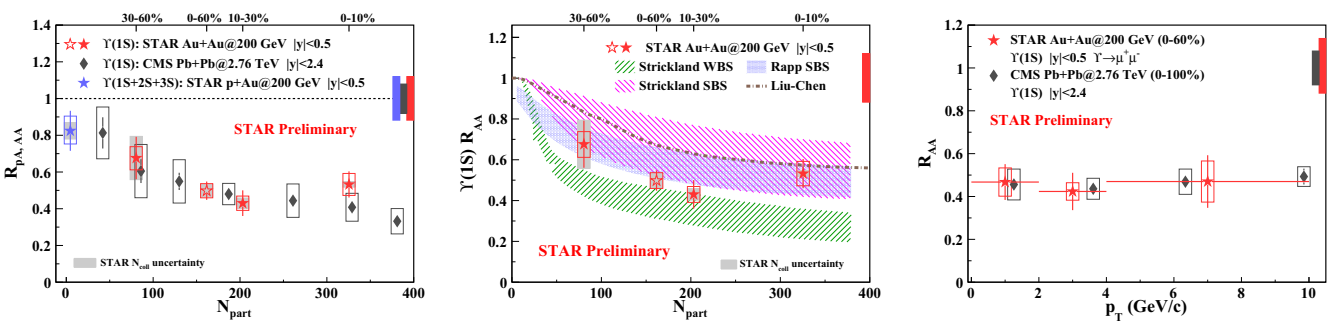

Figure 4. Left: nuclear modification factor, $R_{A A}$, of $\Upsilon(1 S)$ as a function of $N_{\text {part }}$ in Au+Au collisions. Middle: the same data as in the left panel compared to different model calculations. Right: $R_{A A}$ of $\Upsilon(1 S)$ as a function of $p_{T}$ in $0-60 \%$ central $\mathrm{Au}+\mathrm{Au}$ collisions at RHIC and compared to that in $0-100 \%$ central $\mathrm{Pb}+\mathrm{Pb}$ collisions at LHC.
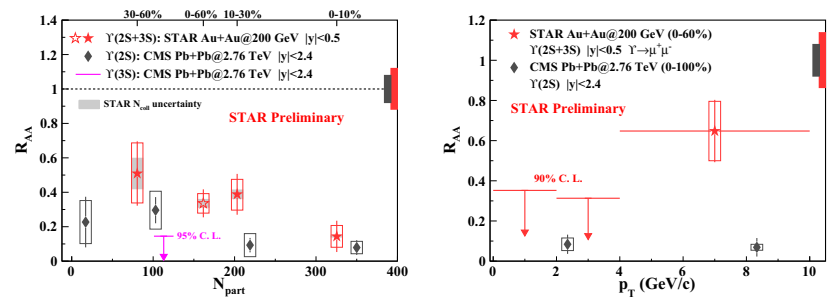

Figure 5. Left: $R_{A A}$ of $\Upsilon(2 S+3 S)$ as a function of $N_{\text {part }}$ in Au+Au collisions. Right: $R_{A A}$ of $\Upsilon(2 S+3 S)$ as a function of $p_{T}$ in $0-60 \%$ central $\mathrm{Au}+\mathrm{Au}$ collisions.

to that from CMS. They show similar suppression and possess no significant $p_{T}$ dependence within uncertainties. The first $\Upsilon(2 S+3 S) R_{A A}$ measurements from STAR as a function of $N_{\text {part }}$ and $p_{T}$ are shown in the left and right panels of Fig. 5, respectively. In comparison with CMS measurements [21], there seems to be a hint of less suppression at RHIC. Also $\Upsilon(2 S+3 S)$ shows a significantly stronger suppression in the most central collisions at RHIC than that for $\Upsilon(1 S)$, which is consistent with the sequential melting phenomenon.

\section{Bulk observables}

Experimentally, the dynamics and the collective behaviour of the sQGP have been studied by measuring the azimuthal anisotropy of the produced particles. Strange hadrons, especially multistrange hadrons, due to their small hadronic interaction cross section and freeze-out temperature closer to the phase transition temperature, are believed to be more sensitive to the partonic stage in heavy-ion collisions. Recent developments indicate that higher order flow harmonics can reveal information about initial state fluctuations, which could help us to better extract the QGP properties. Shown in Fig. 6 are $v_{n}\left(p_{T}\right)$ of $K_{s}^{0}, \phi$ and $\Lambda+\bar{\Lambda}$ at mid-rapidity for $0-10 \%, 10-40 \%$ and $40-80 \%$ central $\mathrm{U}+\mathrm{U}$ collisions at $\sqrt{s_{\mathrm{NN}}}=193 \mathrm{GeV}$ and $\mathrm{Au}+\mathrm{Au}$ collisions at $\sqrt{s_{\mathrm{NN}}}=200 \mathrm{GeV}$ [25]. A clear centrality dependence of $v_{2}\left(p_{T}\right)$ is observed for $K_{s}^{0}, \phi$ and $\Lambda+\bar{\Lambda}$, which is similar to that in $\mathrm{Au}+\mathrm{Au}$ collisions. Higher order flow harmonics $v_{3}$ and $v_{4}$ show much weaker centrality dependence, if any.

New measurements of rapidity-even dipolar flow $\left(v_{1}^{\text {even }}\right)$ from STAR are presented in this conference. These measurements could serve as constraints to distinguish between different initial-state 


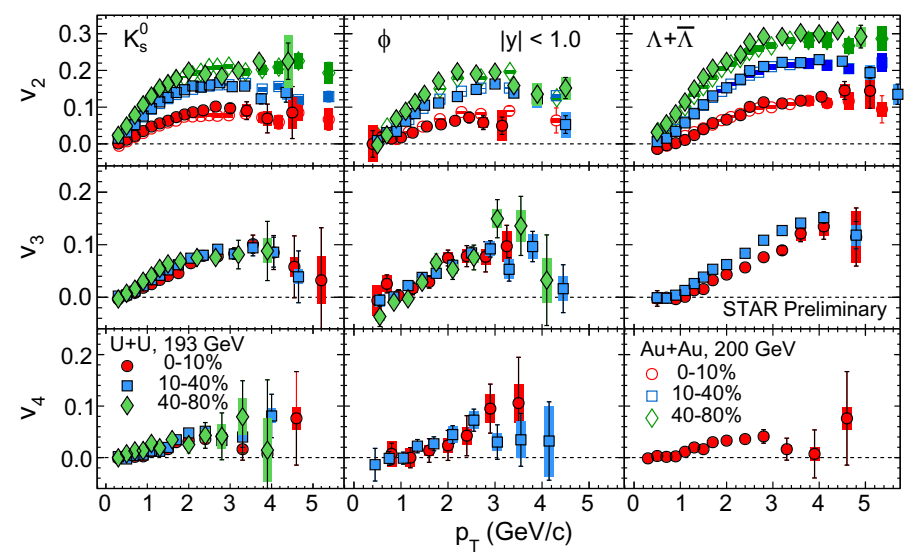

Figure 6. $v_{2}$ (top panels), $v_{3}$ (middle panels) and $v_{4}$ (bottom panels) vs $p_{T}$ of $K_{s}^{0}, \phi$ and $\Lambda+\bar{\Lambda}$ for $0-10 \%, 10-40 \%$ and $40-80 \%$ centrality in $\mathrm{U}+\mathrm{U}$ collisions at $\sqrt{s_{\mathrm{NN}}}=193 \mathrm{GeV}$ and $\mathrm{Au}+\mathrm{Au}$ collisions at $\sqrt{s_{\mathrm{NN}}}=200 \mathrm{GeV}[25]$.

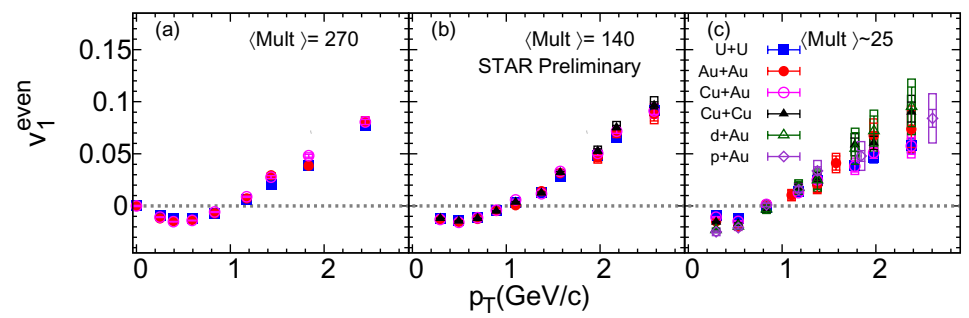

Figure 7. $v_{1}^{\text {even }}$ as a function of $p_{T}$ for different $\left\langle N_{C h}\right\rangle$ selections for different collision systems at $\sqrt{s_{\mathrm{NN}}} \sim 200$ $\mathrm{GeV}$.

models and aid a more reliable extraction of the viscosity over entropy density ratio $\eta / s$. The $v_{1}^{\text {even }}$ for different collision systems are shown in Fig. 7 for different magnitude of $\left\langle N_{c h}\right\rangle$. For each selected $\left\langle N_{c h}\right\rangle$ class, $v_{1}^{\text {even }}\left(p_{T}\right)$ does not show a significant dependence on the collision system, which suggests that the fluctuation-driven initial-state eccentricity $\varepsilon_{1}$ is similar for the six collision systems.

Two-particle interferometry measurement, known as femtoscopy or HBT, is a standard method of studying space-time characteristics of the created system from heavy-ion collisions. Recently collected high-statistic datasets from STAR allow measurements with heavier, but less abundant particles. Fig. 8 shows a comparison of kaon and pion [26] source radii as a function of $m_{T}$ for $0-5 \%$ and $30-40 \% \mathrm{Au}+\mathrm{Au}$ collisions. The $R_{\text {out }}$ values for kaons are systematically larger than those of pions, while the $R_{\text {side }}$ values for kaons and pions are comparable, and the $m_{T}$-dependence of $R_{\text {long }}$ is different between kaons and pions. These observations suggest different space-time configurations at the kinetic freeze-out for the pions and kaons. However, further understanding of the observed behaviors 

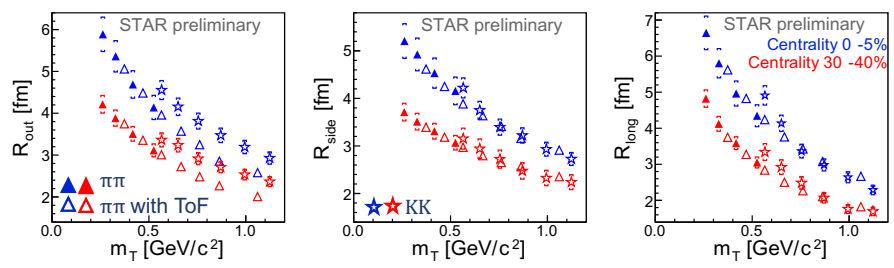

Figure 8. The comparison of the kaon and pion [26] source radii in Au+Au collisions at $\sqrt{s_{\mathrm{NN}}}=200 \mathrm{GeV}$ from STAR.
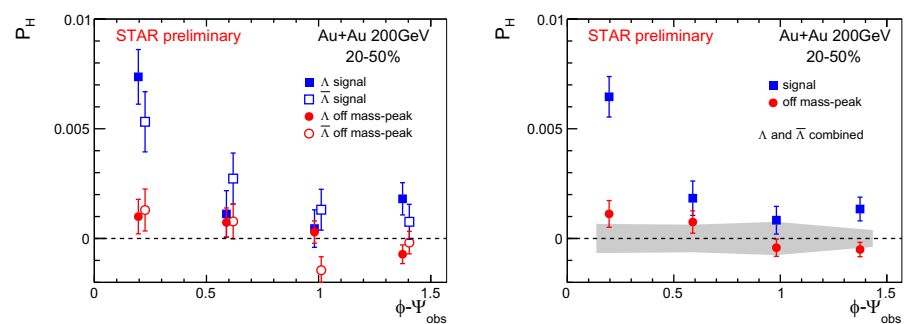

Figure 9. Left: $P_{H}$ for $\Lambda$ and $\bar{\Lambda}$ as a function of $\phi-\Psi_{o b s}$ in $20-50 \%$ central Au+Au collisions at $\sqrt{s_{\mathrm{NN}}}=200 \mathrm{GeV}$. Right: merged $P_{H}$ between $\Lambda$ and $\bar{\Lambda}$ as a function of $\phi-\Psi_{o b s}$ in 20-50\% central Au+Au collisions at $\sqrt{s_{\mathrm{NN}}}=$ $200 \mathrm{GeV}$.

require a comparison with the full three-dimensional hydrodynamic calculations with rescattering in the hadronic phase.

A non-zero global $\Lambda$ polarization has been observed in Au+Au collisions by STAR [27] for the first time, revealing that the matter produced in heavy-ion collisions is by far the most vortical system ever observed. Such vorticity is expected to be maximal at the equator, leads to a larger in-plane than out-of-plane polarization for hyperons. Shown in the left panel of Fig. 9 are the $\Lambda$ and $\bar{\Lambda}$ polarization $\left(P_{H}\right)$ as a function of $\phi-\Psi_{o b s}$ at mid-rapidity in $20-50 \%$ central Au+Au collisions at $\sqrt{s_{\mathrm{NN}}}=200$ $\mathrm{GeV}$, where $\Psi_{o b s}$ is the observed angle of the first-order event plane as an estimate of the reaction plane angle. The right panel of Fig. 9 shows the combined $\Lambda$ and $\bar{\Lambda} P_{H}$ as a function of $\phi-\Psi_{o b s}$. The

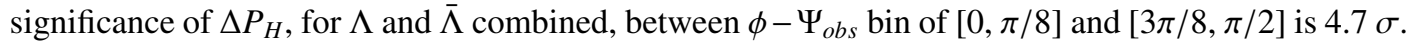
The larger in-plane than out-of-plane polarization is consistent with the picture of maximum vorticity in the equator and low viscosity of the system.

\section{Electro-magnetic probes}

Recently, the ALICE collaboration reported a significant $J / \psi$ excess yield at very low $p_{T}\left(p_{T}<0.3\right.$ $\mathrm{GeV} / \mathrm{c}$ ) in peripheral $\mathrm{Pb}+\mathrm{Pb}$ collisions at $\sqrt{s_{\mathrm{NN}}}=2.76 \mathrm{TeV}$ [28]. This remarkable excess can not be explained by the hadronic production of $J / \psi$ modified by the cold and hot medium effects, however, could be qualitatively explained by coherent photonuclear production mechanism [29]. Shown in the left panel of Fig. 10 are the extracted $J / \psi R_{A A}$ as a function of $p_{T}$ in Au+Au collisions at $\sqrt{s_{\mathrm{NN}}}=$ $200 \mathrm{GeV}$ and $\mathrm{U}+\mathrm{U}$ collisions at $\sqrt{s_{\mathrm{NN}}}=193 \mathrm{GeV}$ from STAR. A huge enhancement of $J / \psi$ production has been observed in peripheral (40-80\%) $\mathrm{Au}+\mathrm{Au}$ and $\mathrm{U}+\mathrm{U}$ collisions for $p_{T}<0.1 \mathrm{GeV} / \mathrm{c}$, 

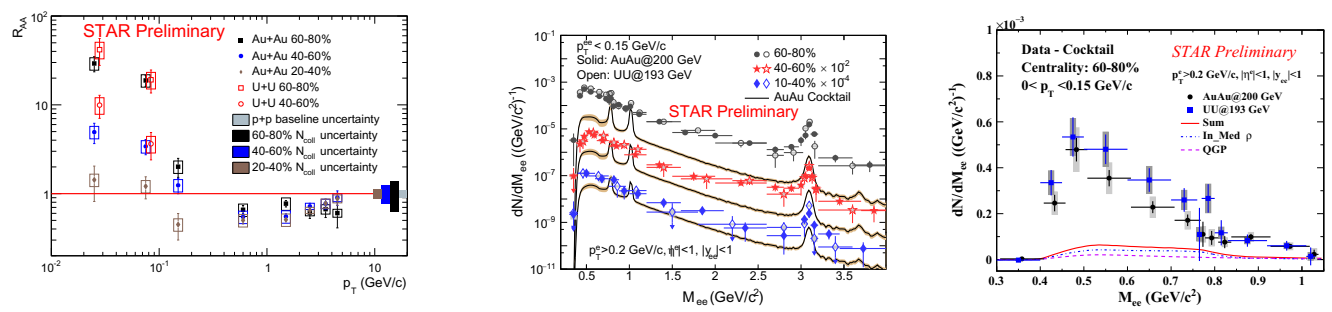

Figure 10. Left: the extracted $J / \psi R_{A A}$ as a function of $p_{T}$ in $\mathrm{Au}+\mathrm{Au}$ collisions at $\sqrt{s_{\mathrm{NN}}}=200 \mathrm{GeV}$ and $\mathrm{U}+\mathrm{U}$ collisions at $\sqrt{s_{\mathrm{NN}}}=193 \mathrm{GeV}$. Middle: centrality dependence of dielectron invariant mass spectrum in the STAR acceptance in $\mathrm{Au}+\mathrm{Au}$ collisions at $\sqrt{s_{\mathrm{NN}}}=200 \mathrm{GeV}$ and $\mathrm{U}+\mathrm{U}$ collisions at $\sqrt{s_{\mathrm{NN}}}=193 \mathrm{GeV}$ for dielectron pair $p_{T}<0.15 \mathrm{GeV} / \mathrm{c}$. The mass spectra are compared to hadronic cocktail simulations shown as solid lines. Right: The low $p_{T}\left(p_{T}<0.15 \mathrm{GeV} / \mathrm{c}\right)$ dielectron excess mass spectra (data-cocktail) within STAR acceptance in $60-80 \%$ collisions, compared with a broadened $\rho$ model calculation.

which confirms the ALICE measurement and points to evidence of coherent photonuclear interaction in hadronic collisions. If indeed, the coherent photonuclear interaction in violent hadronic collisions were the underlying mechanism for the observed $J / \psi$ excess, the coherent photon-photon process would be there and contribute to the dielectron pair production. The middle panel of Fig. 10 shows centrality dependence of dielectron invariant mass spectrum in the STAR acceptance in Au+Au collisions at $\sqrt{s_{\mathrm{NN}}}=200 \mathrm{GeV}$ and $\mathrm{U}+\mathrm{U}$ collisions at $\sqrt{s_{\mathrm{NN}}}=193 \mathrm{GeV}$ for dielectron pairs with $p_{T}<0.15$ $\mathrm{GeV} / \mathrm{c}$. In comparison with the hadronic cocktail calculation, a clear enhancement is observed. The enhancement is larger for more peripheral collisions. To gain more insight into the excess, we subtract the cocktail contributions from the inclusive dielectron data and obtain the excess mass spectra for $p_{T}<0.15 \mathrm{GeV} / \mathrm{c}$ in $60-80 \% \mathrm{~A}+\mathrm{A}$ collisions, as shown in the right panel of Fig. 10. Calculation results from an effective many-body model based on in-medium broadening of the $\rho$-meson spectral function are also shown. The broadened $\rho$ model calculation [30], which has successfully described the dielectron invariant mass spectra integrated over $p_{T}$ at SPS and RHIC, can not describe the significant enhancement yields observed at very low $p_{T}$ in peripheral $\mathrm{A}+\mathrm{A}$ collisions. The enhancement at very low $p_{T}$ measured in peripheral collisions may originate from the coherent photon-photon interactions.

\section{Summary}

At this conference, STAR has presented a wide variety of measurements for $p+p, p+A u, A u+A u$, and $\mathrm{U}+\mathrm{U}$ collisions, at energies ranging from $\sqrt{s_{\mathrm{NN}}}=19.6$ to $200 \mathrm{GeV}$. New results from the HFT include the elliptic and triangular flow of $D^{0}$ mesons and $D_{s} / D^{0}$ and $\Lambda_{c} / D^{0}$ ratios, indicating that charm quarks flow with the medium and hadronize via coalescence mechanism. $J / \psi$ and $\Upsilon$ are measured in the dimuon channel from the MTD to study the dissociation and regeneration of quarkonia in the medium. The dipolar flow, elliptic flow and other higher order flow coefficients are presented to reveal the initial fluctuations and to constrain the transport coefficients of the medium. The source radii of kaon from HBT is presented to probe the space-time evolution of the system. The azimuthal angle dependence of hyperon polarization is studied, which is consistent with the picture of maximum vorticity in the equator and low viscosity of the system. An enhancement of dielectron production at very low $p_{T}$ in peripheral collisions is observed, which may originate from the coherent photoproduction. 


\section{Acknowledgement}

The author was funded by the National Natural Science Foundation of China under Grant Nos. 11505180, 11775213 and 11375172, and MOST under Grant No. 2014CB845400.

\section{References}

[1] P. Braun-Munzinger, J. Stachel, Nature 448, 302 (2007)

[2] A. Beraudo et al., Eur. Phys. J. C 75, 121 (2015)

[3] L. Adamczyk et al. (STAR Collaboration), Phys. Rev. Lett. 118, 212301 (2017)

[4] L.G. Pang et al., Phys. Rev. D 91, 074027 (2015)

[5] G.Q. S. S. Cao, S.A. Bass, Phys. Rev. C 92, 024907 (2015)

[6] M. Nahrgang et al., Phys. Rev. C 91, 014904 (2015)

[7] S. Cao et al., Phys. Rev. C 94, 014909 (2016)

[8] T. Song et al., Phys. Rev. C 92, 014910 (2015)

[9] M. He et al., Phys. Rev. Lett. 110, 112301 (2013)

[10] Y. Oh et al., Phys. Rev. C 79, 044905 (2009)

[11] S. Ghosh et al., Phys. Rev. D 90, 054018 (2014)

[12] V. Greco et al., Phys. Lett. B 595, 202 (2004)

[13] T. Matsui, H. Satz, Physics Letters B 178, 416 (1986)

[14] A. Adare et al. (PHENIX Collaboration), Phys. Rev. C 87, 034904 (2013)

[15] J.P. Lansberg, H.S. Shao, Eur. Phys. J. C 77, 1 (2017)

[16] H.S. Shao, Comput. Phys. Commun. 198, 238 (2016)

[17] E.G. Ferreiro et al., Few Body Syst. 53, 27 (2012)

[18] Y.Q. Ma, R. Vogt, Phys. Rev. D 94, 114029 (2016)

[19] A. Adare et al. (PHENIX Collaboration), Phys. Rev. Lett. 111, 202301 (2013)

[20] X. Zhao, R. Rapp, Phys. Rev. C 82, 064905 (2010)

[21] V. Khachatryan et al. (CMS Collaboration), Phys. Lett. B 770, 357 (2017)

[22] M. Strickland, D. Bazow, Nucl. Phys. A 879, 25 (2012)

[23] Y. Liu et al., Phys. Lett. B 697, 32 (2011)

[24] A. Emerick et al., Eur. Phys. J. A 48, 72 (2012)

[25] B.I. Abelev et al. (STAR Collaboration), Phys. Rev. C 77, 054901 (2008)

[26] L. Adamczyk et al. (STAR Collaboration), Phys. Rev. C 92, 014904 (2015)

[27] L. Adamczyk et al. (STAR Collaboration), Nature 548, 62 (2017)

[28] J. Adam et al. (ALICE Collaboration), Physical Review Letters 116, 222301 (2016)

[29] W. Zha et al. (2017), arXiv: 1705.01460

[30] R. Rapp, Adv. High Energy Phys. 2013, 62 (2013)

[31] A.M. Poskanzer, S.A. Voloshin, Phys. Rev. C 58, 1671 (1998) 